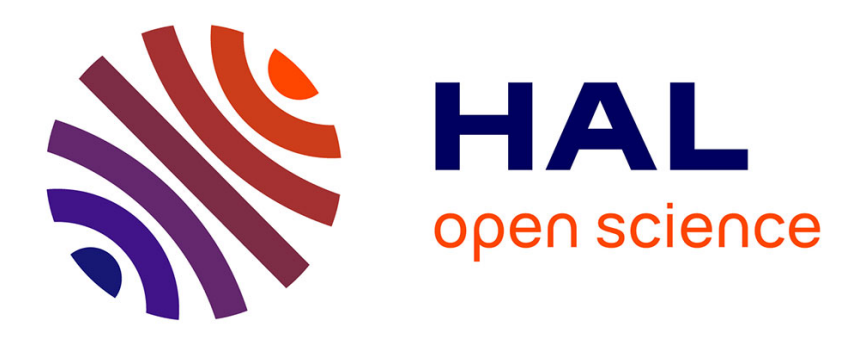

\title{
Production d'impulsions picosecondes et subpicosecondes par lasers à colorant continus à modes bloqués
}

B. Couillaud, A. Ducasse

\section{- To cite this version:}

B. Couillaud, A. Ducasse. Production d'impulsions picosecondes et subpicosecondes par lasers à colorant continus à modes bloqués. Revue de Physique Appliquée, 1979, 14 (2), pp.331-345. 10.1051/rphysap:01979001402033100 . jpa-00244602

\section{HAL Id: jpa-00244602 https://hal.science/jpa-00244602}

Submitted on 1 Jan 1979

HAL is a multi-disciplinary open access archive for the deposit and dissemination of scientific research documents, whether they are published or not. The documents may come from teaching and research institutions in France or abroad, or from public or private research centers.
L'archive ouverte pluridisciplinaire HAL, est destinée au dépôt et à la diffusion de documents scientifiques de niveau recherche, publiés ou non, émanant des établissements d'enseignement et de recherche français ou étrangers, des laboratoires publics ou privés. 


\title{
Production d'impulsions picosecondes et subpicosecondes par lasers à colorant continus à modes bloqués
}

\author{
B. Couillaud et A. Ducasse \\ Centre de Physique Moléculaire, Optique et Hertzienne $\left({ }^{*}\right)$ \\ Université de Bordeaux-I, 33405 Talence, Francé \\ (Reçu le 30 juin 1978, révisé le 16 octobre 1978, accepté le 8 novembre 1978)
}

\begin{abstract}
Résumé. - Une analyse du fonctionnement d'un laser en régime modes bloqués, permettant la production d'impulsions picosecondes, est présentée ; on insiste particulièrement sur le cas des lasers à colorant continus, seuls capables de délivrer des impulsions subpicosecondes. On montre tout d'abord la nécessité de bloquer entre elles les phases des modes d'un laser pour obtenir une concentration de l'énergie dans le temps. Les différentes méthodes de blocage sont alors analysées à l'aide d'images et de considérations qualitatives très simples. Les principaux lasers picosecondes à colorant sont décrits à titre d'application de ces méthodes. Après avoir indiqué comment l'on mesure les durées d'impulsions, on compare leurs performances à celles d'autres types de lasers picosecondes. Enfin on donne en conclusion une idée des développements les plus récents des recherches dans ce domaine.
\end{abstract}

\begin{abstract}
An analysis of the operation of mode-locked lasers producing picosecond pulses is given, with emphasis on the CW dye laser which is alone capable of delivering subpicosecond pulses. First, it is shown how the locking of the phases of the modes of a laser is necessary for the compression of light energy into a short time interval. Then, the various methods of mode-locking are analyzed with the aid of very simple qualitative descriptions and physical pictures. Experimental realizations of each method are also given. After describing how the pulse durations are measured, the performances of the various picosecond laser systems are compared. By way of conclusion, a few brief ideas of the most recently research developments in the field are given.
\end{abstract}

Dans cet article de revue s'adressant aux nonspécialistes, on se propose de faire le point sur les possibilités actuelles de concentration de l'énergie lumineuse dans le temps, grâce aux lasers à modes bloqués. On s'intéressera plus particulièrement aux lasers à colorant continus à modes bloqués, tout en donnant des notions de base et en comparant les performances obtenues avec les différents systèmes lasers de production d'impulsions picosecondes et subpicosecondes. Pour rendre la lecture aisée à tous, les notions les plus élémentaires seront rappelées; d'autre part des simplifications, souvent grossières, seront faites pour présenter les notions complexes, dans le but, généralement, de donner au physicien non-spécialiste une image à peu près correcte des processus. Aucune tentative ne sera faite ici pour traiter les problèmes rigoureusement; on renvoie les lecteurs plus exigeants aux articles et livres de base à usage des spécialistes qui sont cités en références [1-5].

(*) Laboratoire associé au C.N.R.S.
Après avoir montré comment la distribution temporelle de l'intensité de sortie d'un laser est fonction du blocage des phases de ses modes et indiqué comment ceux-ci se couplent, on donnera les méthodes conduisant au fonctionnement modes bloqués. On insistera sur la méthode de blocage par absorbant saturable qui est à la fois la plus difficile à analyser, la plus difficile à mettre en ouvre, mais aussi la plus efficace, celle qui conduit actuellement aux meilleurs résultats. Après avoir rapidement évoqué le problème fondamental de la mesure des longueurs des impulsions picosecondes et subpicosecondes, on commentera un tableau des performances des sources le plus couramment utilisées. On remarquera, lors de cette comparaison, que les lasers à colorant continus et modes bloqués ont encore un assez faible domaine d'accord en longueur d'onde par rapport à leurs possibilités. On consacrera alors la dernière partie à la description des efforts qui sont faits actuellement, et auxquels nous participons, pour étendre cette plage.

1. Blocage des modes et distribution temporelle de l'intensité de sortie d'un laser. - La distribution en 
fréquence de l'émission d'un laser est généralement constituée d'un certain nombre de fréquences discrètes. Chacune de ces fréquences correspond à un mode de résonance de la cavité laser : longueur $L$ de la cavité égale à un multiple entier, $k+1$, de la demi-longueur d'onde $\lambda_{k} / 2$; soit encore la condition suivante sur la fréquence $v_{k}$ correspondante :

$v_{k}=(k+1) \frac{c}{2 L}$.

Ces modes sont appelés modes longitudinaux du laser. Ils sont sélectionnés à partir de l'ensemble des modes résonnants de la cavité par la relation évidente devant exister entre le gain du milieu amplificateur et les pertes de la cavité (Fig. 1) : seuls ceux pour lesquels le gain du milieu amplificateur est supérieur aux pertes de la cavité, peuvent donner naissance à l'effet laser. La distribution correspondante dans le temps de l'émission du laser, résulte de la superposition des champs électriques $\mathbf{E}_{\boldsymbol{k}}$ de ces différents modes longitudinaux de fréquences très voisines. Elle dépend de leurs amplitudes, de leurs phases relatives et de leurs polarisations. Consi-
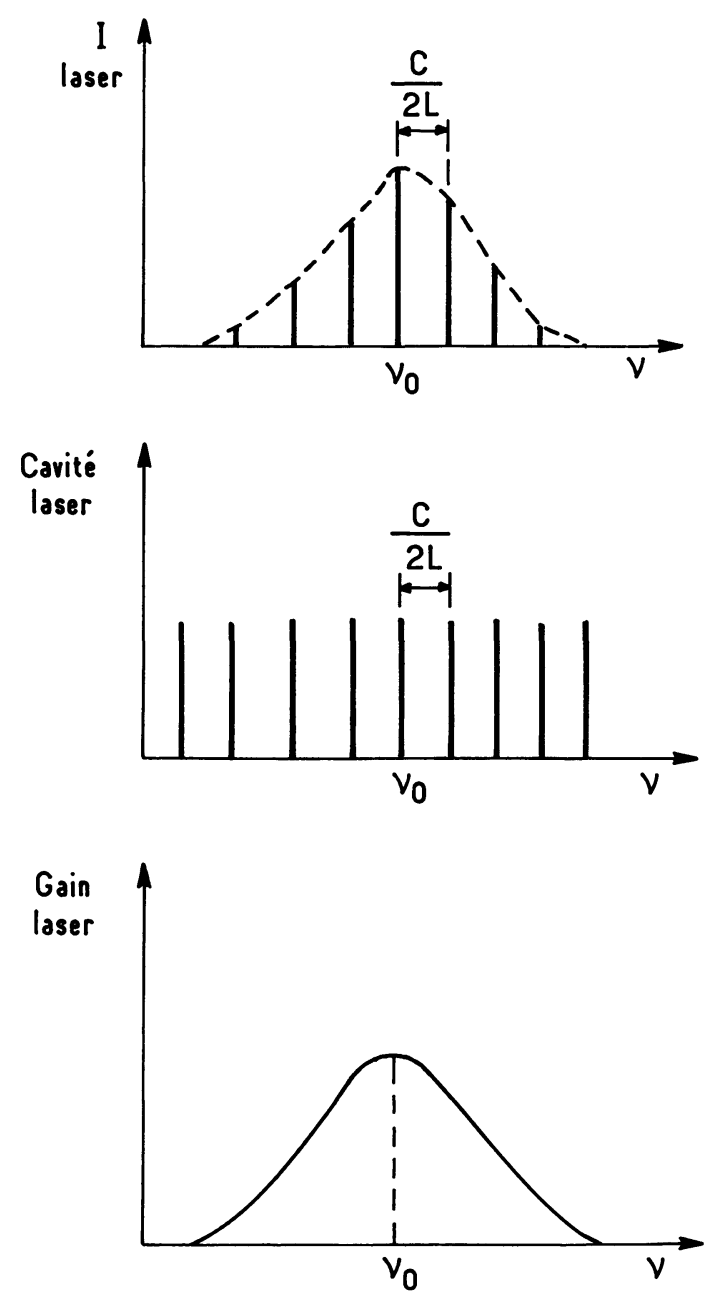

Fig. 1. - Modes longitudinaux d'un laser. $1 a$ Modes longitudinaux du laser. $1 b$ Modes de la cavité. $1 c$ Courbe de gain du laser. dérons, pour schématiser le problème, le cas où seulement deux modes voisins de fréquences $v_{k}$, $v_{k+1}$ ayant même polarisation, même amplitude $a$ et des phases $\varphi_{k}, \varphi_{k+1}$ quelconques coexistent :

$$
\begin{aligned}
& E_{k}=a \cos \left(2 \pi v_{k} t+\varphi_{k}\right) \\
& E_{k+1}=a \cos \left(2 \pi v_{k+1} t+\varphi_{k+1}\right) .
\end{aligned}
$$

Les détecteurs de signaux optiques ont des temps de réponse $\theta$ ne leur permettant pas de suivre les oscillations aux fréquences optiques $v_{k}$ (de l'ordre de $5 \times 10^{14} \mathrm{~Hz}$ ). On définit alors une intensité moyenne $I(t)$, proportionnelle à la moyenne sur une période du carré du champ total $E=E_{k}+E_{k+1}$ :

$$
\begin{aligned}
I(t) & =\alpha\left\langle E^{2}\right\rangle \\
& =\alpha a^{2}\left|1+\cos \left(2 \pi\left(v_{k+1}-v_{k}\right) t+\varphi_{k+1}-\varphi_{k}\right)\right|
\end{aligned}
$$

Au spectre $I(v)$ composé de deux ondes monochromatiques $v_{k}, v_{k+1}$, correspond donc une distribution d'intensité $I(t)$ modulée sinusoïdalement à la fréquence $\left(v_{k+1}-v_{k}\right)$ Fig. (2). Ce phénomène de battement entre deux fréquences voisines est bien connu dans le domaine des ondes sonores où il est d'observation courante. Dans le domaine optique, cependant, se pose le problème de la cohérence des champs $E_{k}$ et $E_{k+1}$ : l'approximation, faite précédemment, consistant à décrire $E_{k}$ par une onde purement monochromatique et donc de fréquence $v_{k}$ et de phase $\varphi_{k}$ indépendantes du temps, n'a de sens que si elle peut être considérée comme valide sur des temps très grands par rapport au temps de réponse de la détection. C'est vrai en acoustique; c'est le plus souvent faux pour les modes d'un laser. Leur comportement réel, non monochromatique, très difficile à prendre en compte correctement, peut être schématisé de la manière suivante : la fréquence $v_{k}$ reste indépendante du temps, mais $\varphi_{k}$ subit des fluctuations aléatoires, dont le temps caractéristique $\theta_{c}$ (par exemple de l'ordre de $100 \mathrm{~ns}$ ) est fonction de la stabilité de la source. Si les phases $\varphi_{k}$ et $\varphi_{k+1}$ varient indépendamment l'une de l'autre, lorsque l'on observe l'intensité $I(t)$ sur des temps très longs par rapport i $\theta_{\mathrm{c}}$, le terme d'interférences des deux champs : $\cos \left|2 \pi\left(v_{k+1}-v_{k}\right) t+\varphi_{k+1}-\varphi_{k}\right|$ se trouve moyenné à 0 en raison des valeurs aléatoires prises par la différence des phases durant le temps d'observation. On enregistre alors une intensité constante égale à
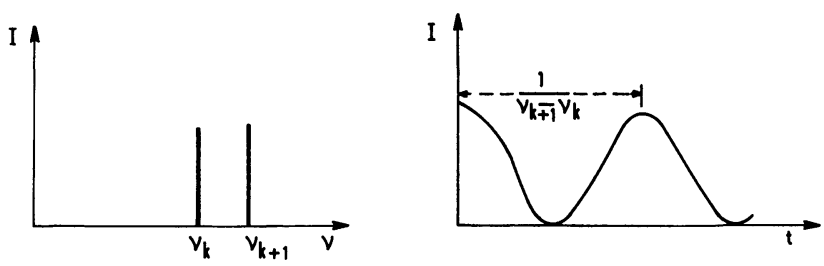

Fig. 2. - Battement entre deux ondes monochromatiques de fréquences voisines. 
la somme des intensités des deux modes. Pour obtenir un battement lumineux on est donc conduit à trouver une méthode qui permette de fixer l'une des phases par rapport à l'autre : on cherche à bloquer les modes en phase.

On peut généraliser les considérations précédentes au cas du laser fonctionnant sur $\mathrm{n}$ modes longitudinaux. Si les phases $\varphi_{k}$ de ces modes varient aléatoirement indépendamment les unes des autres, les termes d'interférences entre modes donnent une valeur moyenne nulle sur le temps de réponse $\theta$ de la détection, si $\theta$ est très supérieur à $\theta_{\mathrm{c}}$. L'intensité enregistrée à la sortie du laser est alors la somme constante des intensités des différents modes. C'est le cas pour le laser dit libre c'est-à-dire sans artifices pour bloquer les phases entre elles, et pour lequel les modes longitudinaux sont généralement assez peu couplés entre eux. Si l'on réussit à fixer les phases entre elles, alors l'intensité $I(t)$ dépend des termes d'interférences qui sont fonctions des amplitudes et phases relatives des différents modes. Lorsque l'on connait la distribution des amplitudes et des phases sur tout le spectre $I(v)$, la transformation de Fourier permet de déterminer sans ambiguïté $I(t)$. Une situation particulièrement intéressante, pouvant être réalisée expérimentalement, est celle dans laquelle tous les modes ont la même phase $\varphi_{k}=\varphi_{0}$. A une distribution $I(v)$ de modes $v_{k}$ distants de $\delta v=c / 2 L$ et occupant un domaine spectral $\Delta v$, correspond une distribution $I(t)$ formée d'impulsions, de durées $\tau$ à peu près égales à $1 / \Delta v$ et de période $T=\frac{1}{\delta v}=\frac{2 L}{c}$ (Fig. 3 ). On constate

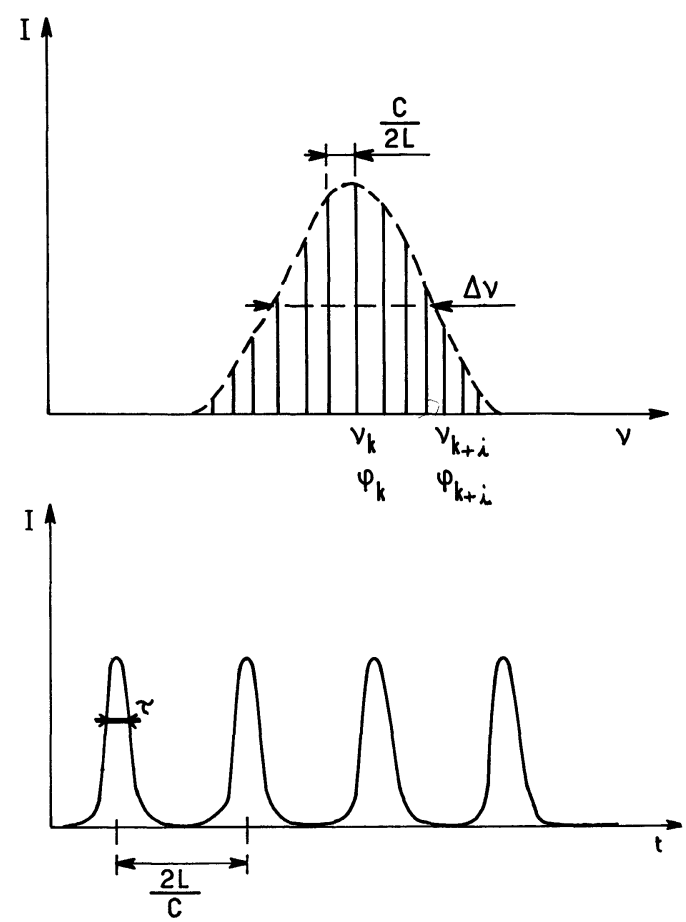

Fig. 3. - Correspondance entre distribution spectrale $I(v)$ et distribution temporelle $I(t)$ lorsque toutes les phases $\varphi_{k}$ sont égales. donc qu'en bloquant les modes d'un laser, on est capable de concentrer son énergie dans des impulsions d'autant plus brèves que la bande spectrale occupée est plus large. Comme l'on connaît les bandes spectrales correspondant aux courbes de gain pour les différents types classiques de laser, on peut dès maintenant comparer leurs possibilités théoriques quant à la production d'impulsions très courtes : l'élargissement Doppler détermine la bande $\Delta v$ disponible pour les lasers à gaz : $\Delta v_{\text {gaz }}$ est au maximum égal à $10^{10} \mathrm{~Hz}\left(10^{-1} \AA\right)$ conduisant à une durée d'impulsions idéales $\tau \simeq 100$ ps; pour les lasers à solide, par exemple à verres dopés, la largeur de bande $\Delta v_{\text {verres dopés }} \simeq 10^{12} \mathrm{~Hz}(10 \AA)$ conduit à $\tau \simeq 1 \mathrm{ps}$; pour les lasers à liquides (à colorant) $\Delta v_{\text {colorant }} \simeq 10^{13} \mathrm{~Hz}(100 \AA)$, soit $\tau \simeq 0,1$ ps. Les lasers à colorant seront donc $a$ priori les seuls candidats possibles pour l'obtention d'impulsions subpicosecondes.

On a, dans ce qui précède, analysé la distribution temporelle de l'intensité de sortie d'un laser supposé à modes bloqués; il est intéressant d'expliciter la distribution spatiale correspondante à l'intérieur de la cavité de l'oscillateur. Si l'on se reporte à la figure 4, on voit que l'extraction d'une partie de l'énergie interne à l'oscillateur se fait par l'intermédiaire du miroir $\mathbf{M}_{2}$ dont la transparence a été convenablement ajustée. La production d'impulsions à la fréquence $c / 2 L$ correspond donc à la présence d'un train d'onde de longueur $\Delta l=c / \tau=c \Delta v$ se propageant à la vitesse $c$ à l'intérieur de la cavité. Chaque fois que ce train d'onde arrive sur $\mathbf{M}_{2}$, celui-ci transmet une faible partie de son énergie à l'extérieur, construisant ainsi un train d'impulsions distantes de $2 L$ dans l'espace, caractéristique de la sortie du laser.

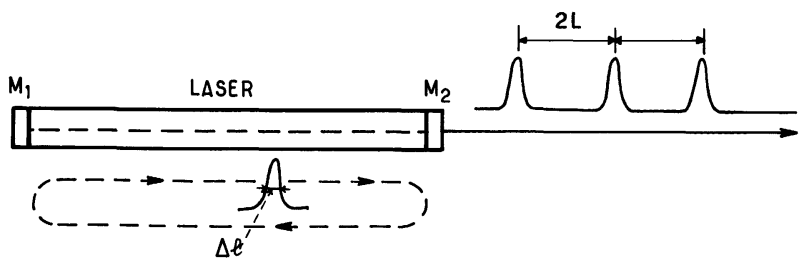

Fig. 4. - Propagation d'un train d'onde à l'intérieur du laser.

Avec une impulsion de $1 \mathrm{ps}$, la longueur $\Delta l$ est égale à $0,3 \mathrm{~mm}$; l'énergie lumineuse n'est plus à̀ un instant donné distribuée de manière homogène le long de la cavité, comme dans le laser en régime libre.

Remarque. - Blocage des modes transverses d'un laser. Nous avons supposé implicitement dans tout ce qui précède, que le laser étudié fonctionne sur un mode transverse unique, et n'avons donc envisagé que les couplages entre modes longitudinaux appartenant à celui-ci. On montre toutefois, lors de l'étude des cavités ouvertes utilisées comme résonateurs pour les oscillateurs laser [6], que la recherche des états stationnaires du champ à l'intérieur de ces 
dernières, conduit à trouver une infinité de solutions caractérisées chacune par une distribution transverse (dans un plan perpendiculaire à l'axe) particulière de l'intensité. Chacune de ces distributions est caractéristique de ce que l'on a appelé un mode transverse. L'état stationnaire du champ est alors parfaitement défini lorsque l'on a associé à chacun de ces modes transverses l'ensemble des modes longitudinaux précédemment décrits et définis à partir de la condition de résonance $L=(k+1) \lambda_{k} / 2$. La plupart des lasers (commerciaux) fonctionnent sur le mode transverse fondamental $\mathrm{TEM}_{00}$, auquel correspond une distribution de l'intensité gaussienne par rapport à l'axe. Il est cependant facile de faire apparaître d'autres modes transverses en défavorisant, par un léger déréglage des miroirs, le mode TEM $_{00}$ de symétrie la plus élevée. Dans une configuration déterminée de la cavité (favorisant l'apparition des modes transverses), en raison de couplages sur lesquels on reviendra plus tard, seul un petit nombre de ceux-ci peuvent coexister. Lorsque l'on se place dans une situation comportant plusieurs modes transverses, l'analyse est compliquée en raison du nombre important de modes susceptibles de se coupler : les modes longitudinaux de chaque mode transverse peuvent, par exemple, se coupler, indépendamment des autres modes transverses, pour donner naissance à une impulsion; on obtient alors un fonctionnement où coexistent dans la cavité autant d'impulsions que de modes transverses [7]. Mais on peut aussi avoir un couplage entre les modes longitudinaux correspondant par exemple à la même valeur $k$ mais appartenant à différents modes transverses [8]; on peut alors aboutir au résultat original suivant : l'axe du faisceau ne conserve pas une direction fixe dans l'espace.

Ce balayage spatial très rapide a pu être mis en évidence en enregistrant, au moyen d'une photodiode de très faible dimension placée au voisinage de la position moyenne de l'axe du faisceau, les variations d'intensité engendrées par le déplacement de celui-ci. Cependant, les applications actuelles du blocage des modes transverses sont peu importantes, surtout en raison d'un contrôle très difficile des couplages. La suite de l'article concernera uniquement le blocage des modes longitudinaux.

2. Saturation d'un milieu par une onde et couplages entre modes. - Afin d'étudier les méthodes conduisant au blocage des phases désiré, nous allons d'abord montrer comment les différents modes peuvent se coupler. Ce couplage ne pouvant se produire que par l'intermédiaire du milieu avec lequel ils interagissent, on est conduit à faire une analyse qualitative très succincte de l'interaction onde-milieu.

Lorsqu'une onde électromagnétique monochromatique (un seul mode) interagit avec un milieu, cela se traduit entre autres par son absorption ou son amplification; en retour, si elle est suffisamment intense, elle tend (si l'on veut donner une image simple à deux niveaux), à rendre égales les populations des niveaux inférieur et supérieur de la transition. On dit que l'onde électromagnétique sature le milieu. Cet effet de saturation est bien entendu d'autant plus important que l'intensité du mode est grande.

Si l'on envoie, dans le milieu saturé par un premier mode longitudinal un deuxième mode de fréquence très voisine, celui-ci voit un milieu perturbé par le premier, tout au moins lorsque les deux modes interagissent avec les mêmes atomes ou molécules, ce qui est le cas lorsque l'écart en fréquences entre les deux modes considérés est inférieur à la largeur spectrale de la transition impliquée (largeur homogène de la transition) $\left({ }^{1}\right)$. Il est bien évident que deux modes voisins devant chercher leur gain parmi un ensemble commun de molécules, ils n'auront pas le même comportement que s'ils existaient seuls séparément dans le milieu. La nécessité de se partager le gain du milieu amplificateur conduit à un couplage entre les deux modes. Toute variation de l'amplitude ou de la phase d'un des modes, va induire des variations sur le second ; on dit que les deux modes sont couplés par l'interaction non linéaire onde-milieu.

Chaque mode longitudinal forme une onde stationnaire à l'intérieur de la cavité laser. La saturation du milieu considéré par un mode, a la même distribution que le carré de son champ : elle présente des maxima (ventres de champ) et des minima (nœuds de champ) distants de $\lambda / 2$. Le couplage, précédemment décrit, sera d'autant plus efficace que, à l'intérieur du milieu, les maxima de champ d'un des modes coïncident avec les maxima de l'autre. On peut jouer sur cette distribution spatiale de la saturation pour faire varier le couplage des modes longitudinaux d'un laser fonctionnant en régime libre. Cependant, comme le suggèrent les considérations simples précédentes, il sera toujours impossible de supprimer complètement les couplages entre modes d'un laser en fonctionnement multimode.

3. Méthodes de blocage des modes. - En fait, le problème, pour concentrer l'énergie dans le temps, n'est pas de supprimer les couplages entre modes, mais au contraire, comme on l'a vu, de les favoriser et surtout de les contrôler. Plusieurs méthodes ont été proposées, que l'on passera en revue, en insistant surtout sur celles conduisant aux applications les plus importantes.

3.1 Auto-Blocage. - On peut simplement, en utilisant judicieusement les couplages préexistants dans le milieu amplificateur et que l'on vient d'évoquer, obtenir le blocage des phases. Cet effet d'autoblocage a été largement étudié théoriquement (voir par exemple [10a]) et obtenu expérimentalement dans

( $\left.{ }^{1}\right)$ Pour une définition des élargissements homogènes ou inhomogènes, voir par exemple [9]. 
le cas des principaux lasers à gaz et à solides. Cependant, on contrôle généralement mal le blocage et la sortie pulsée que l'on obtient est le plus souvent instable; cette méthode semble avoir été à peu près abandonnée pour la production d'impulsions. On préfère maintenant imposer aux modes un blocage de phases par une modification des conditions de fonctionnement du laser, soit commandée de l'extérieur, c'est la méthode active, soit par simple introduction d'un élément passif dans la cavité, c'est la méthode passive.

3.2 Blocage actif. - 3.2.1 Principe. - On suppose que l'on sait moduler sinusoïdalement à une fréquence $f$ déterminée l'amplitude d'un des modes de fréquence $v_{k}$ du laser. Son spectre en fréquence a alors une composante à la fréquence centrale $v_{k}$ et deux bandes latérales à $v_{k} \pm f$ (Fig. 5).
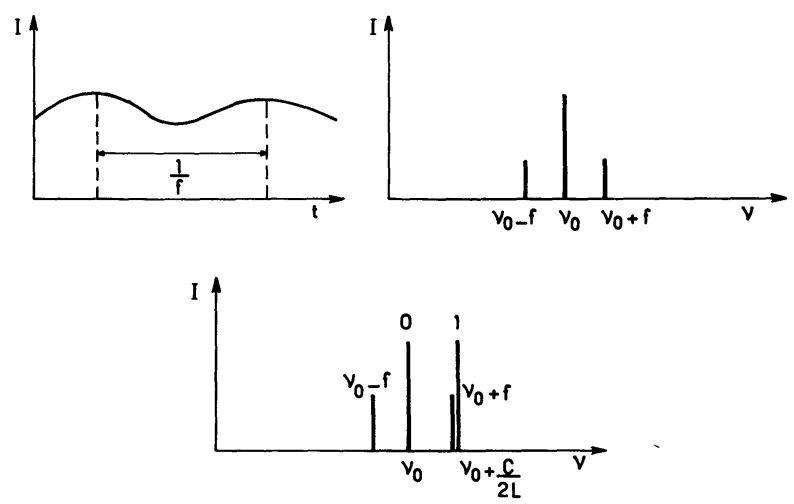

Fig. 5. - Production de bandes latérales par modulation de l'amplitude d'un mode. $5 a$ Modulation de l'amplitude d'un mode du laser. $5 b$ Spectre de cette émission modulée. $5 c$ Superposition de ce spectre et de celui du mode voisin.

Son couplage avec le mode voisin de fréquence $v_{k+1}=v_{k}+\frac{c}{2 L}$ devient d'autant plus fort que la fréquence $v_{k}+f$ de sa bande latérale est plus voisine de $v_{k+1}$, soit que $f$ est plus voisin de $c / 2 L$. Ce couplage a un rôle de synchronisation vis-à-vis du mode $k+1$ : celui-ci passe d'un régime d'oscillations quasi libres (en l'absence de modulations) à un régime d'oscillations forcées par la bande latérale à la fréquence $v_{k}+f$; le mode $k+1$ et la bande latérale tendent à osciller à la même fréquence et en phase l'un avec l'autre. Cette réaction est à rapprocher du résultat obtenu, en mécanique, lorsque l'on couple fortement deux pendules dont les périodes propres d'oscillation sont voisines : ils se synchronisent et se mettent à osciller en phase.

Si l'on module, en fait, l'amplitude de l'ensemble des modes longitudinaux, chaque mode se trouve bloqué en phase et en fréquence par une bande latérale du mode voisin. Les phases de tous ces modes qui oscillaient presque librement dans la bande du gain saturé du laser, se fixent les unes par rapport aux autres et l'on tend vers une distribution d'intensité correspondant dans la cavité à une impulsion dont la durée est de l'ordre de $1 / \Delta v, \Delta v$ représentant la largeur de bande du gain non saturé (Fig. 6).
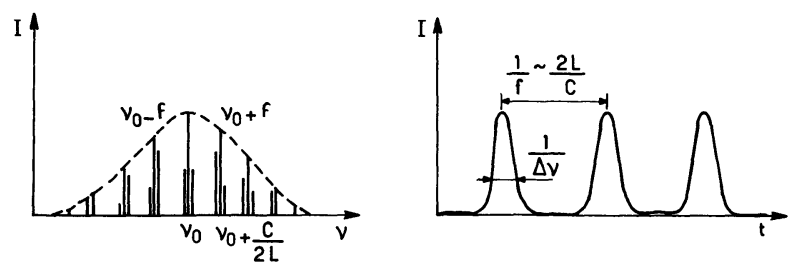

Fig. 6. - Blocage des phases des modes par modulation à la fréquence $c / 2 L$ de leurs amplitudes.

En effet, entre deux passages de l'impulsion à travers le milieu amplificateur, ce dernier a généralement le temps (cas des lasers à colorant par exemple) de reconstruire l'inversion de population existant en absence de radiation laser. Le milieu va donc présenter, à l'arrivée de l'impulsion, un gain non saturé. Ce gain est bien entendu supérieur à celui que l'on obtient dans le cas d'un fonctionnement continu de l'oscillateur, puisque l'absence des effets de saturation avant passage de l'impulsion, conduit à créer une différence de population beaucoup plus grande que celle obtenue en régime continu. On peut aussi remarquer que, suivant la nature de l'élargissement (homogène ou inhomogène [9]) du milieu amplificateur, la largeur spectrale de la bande de gain sera ou non sensible aux effets de saturation. Dans le cas d'un élargissement inhomogène, cette largeur est à peu près la même, que le milieu soit saturé ou non. Aussi la connaissance de cette dernière en régime saturé (fonctionnement continu de l'oscillateur) permet-elle de prédire pour un laser donné la largeur minimale des impulsions que l'on peut obtenir en régime modes bloqués à partir de celui-ci. Dans le cas d'un élargissement homogène, la largeur spectrale de la courbe de gain est fonction de la saturation du milieu, et diminue avec celle-ci.

Ceci explique, par exemple, que dans le cas des lasers à colorant continus, la bande de gain est beaucoup plus étroite en régime continu de fonctionnement de l'oscillateur (forte saturation) que dans le cas du fonctionnement en modes bloqués, le gain vu par le train d'onde à l'intérieur de la cavité laser étant alors essentiellement non saturé. Pour une configuration donnée de laser à colorant, on passe d'une largeur spectrale d'émission de $0,2 \AA$ en régime continu à plusieurs $\AA$ pour le même système fonctionnant en modes bloqués.

On peut aussi bloquer les modes en modulant, non plus leur amplitude, mais leur phase (ou, ce qui est équivalent, leur fréquence). Il suffit d'introduire dans la cavité un élément permettant une modulation de l'indice. Cette méthode de blocage actif, qui a été expérimentée dans le cas de lasers à solides [10b] est sélective par rapport à la fréquence et n'a pas été 
utilisée pour la production d'impulsions de l'ordre de la picoseconde. On se contentera donc de présenter la méthode précédente.

La modulation de l'amplitude des modes peut être obtenue de deux manières différentes : cavité,

- on module à une fréquence $f$ les pertes de la

- on module à $f$ le gain du milieu amplificateur.

3.2.2 Réalisation. - On décrit pour illustrer cette double possibilité un seul montage dans lequel les deux méthodes précédentes sont successivement utilisées : le laser à colorant pompé en synchronisme par un laser $\mathrm{Ar}^{+}$modes bloqués. Dans le laser ionique représenté en figure 7, le prisme a non seulement son rôle classique de sélecteur de raies, mais aussi le rôle de modulateur des pertes de la cavité. Une onde ultra-sonore, produite à partir d'un élément piézo-électrique, provoque dans le prisme de quartz, par effet acousto-optique, une modulation de l'indice de réfraction à la fréquence $f$, que l'on choisit égale à $c / 2 L_{1}$, lorsque l'on désigne par $L_{1}$ la longueur de la cavité du laser $\mathrm{Ar}^{+}$. Cette modulation de l'indice induit la modulation désirée des pertes. Le laser $\mathrm{Ar}^{+}$ fonctionne alors en régime modes bloqués; il produit un train continu d'impulsions d'une durée $\tau$ de l'ordre de 0,1 à $1 \mathrm{~ns}$, distantes de $T \simeq 10 \mathrm{~ns}$. Typiquement, on peut obtenir des puissances crêtes de l'ordre de $100 \mathrm{~W}$.

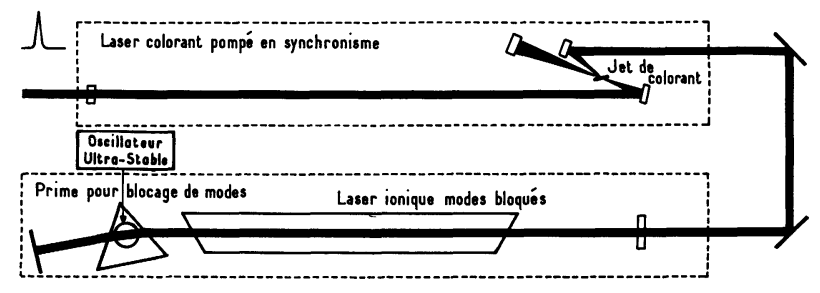

Fig. 7. - Laser à colorant continu, modes bloqués par un pompage synchrone produit par un laser $\mathrm{Ar}^{+}$modes bloqués (d'après un dispositif commercialisé par Spectra-Physics).

Le laser $\mathrm{Ar}^{+}$à modes bloqués, dans le montage représenté en figure 7 , sert à pomper un laser à colorant. Le milieu amplificateur du laser à colorant, au lieu de recevoir l'énergie constante d'un laser $\mathrm{Ar}^{+}$ continu, reçoit le train continu d'impulsions décrit précédemment. Son gain est modulé à la fréquence $f=c / 2 L_{1}$; si la distance entre modes longitudinaux du laser à colorant $c / 2 L_{2}\left(L_{2}\right.$ longueur de la cavité du colorant) est égale à $f$, le laser à colorant fonctionne, lui aussi, en régime modes bloqués; on dit qu'il est pompé en synchronisme par le laser $\mathrm{Ar}^{+}$. Il suffit pour cela de réaliser l'égalité des longueurs $L_{1} \simeq L_{2}$. On obtient encore un train continu d'impulsions de durées de l'ordre de $1 \mathrm{ps}$, à la même fréquence de répétition $(\simeq 100 \mathrm{MHz})$ bien entendu, que dans le train de pompage. Les puissances crêtes disponibles peuvent être de plusieurs centaines de watts. Outre la réduction de la durée des impulsions et l'augmentation de la puissance crête, l'utilisation du laser à colorant permet un accord de la longueur d'onde centrale du train d'impulsions sur toute la bande d'accord du colorant pompé, grâce à un élément dispersif non représenté en figure 7.

\subsection{Blocage Passif. - 3.3.1 Principe. - On} peut obtenir aussi un blocage efficace des modes d'un laser, sans modulation commandée de l'extérieur à la fréquence $c / 2 L$, à condition d'introduire dans la cavité du laser, comprenant déjà le résonateur et le milieu amplificateur, un milieu absorbant saturable; le rôle de ce troisième élément, si l'on veut le schématiser très grossièrement, consiste à apporter dans la cavité des pertes à la fréquence $c / 2 L$ existant entre modes longitudinaux.

Il faut tout d'abord définir ce qu'est un absorbant saturable pour une expérience donnée : on appelle absorbant saturable tout matériau dont le coefficient d'absorption décroît notablement lorsque l'on fait croître l'intensité lumineuse qui le traverse de zéro à l'intensité maximale disponible. Cette définition peut être traduite concrètement par la courbe (Fig. 8) donnant la transmission de l'absorbant saturable en fonction de l'intensité lumineuse $I$ à laquelle il est soumis : pour de très faibles intensités, l'absorbant saturable a un fort coefficient d'absorption et ne transmet qu'une faible partie de l'intensité incidente ; le coefficient d'absorption décroît, cependant, au fur et à mesure que $I$ augmente pour être pratiquement nul pour des valeurs de $I$ supérieures à une valeur $I_{\mathrm{A}}$ que l'on désigne sous le nom d'intensité de saturation et qui est une caractéristique de l'absorbant. Celui-ci est alors pratiquement transparent, sa transmission est égale à 1 .

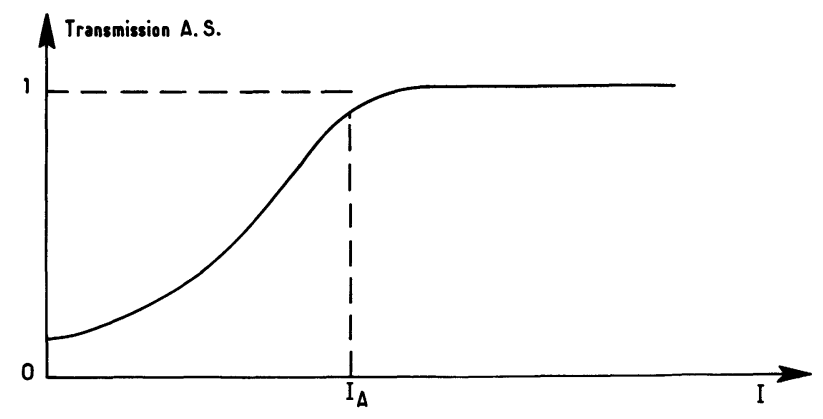

Fig. 8. - Variations de la transmission d'un absorbant saturable.

Ce comportement peut être facilement interprété en termes simples d'interaction onde-milieu : on a remarqué précédemment que l'onde absorbée par un milieu le sature toujours; tant que l'intensité $I$ de l'onde est très inférieure à $I_{\mathrm{A}}$, la modification à l'équilibre des populations qu'elle provoque est faible et le coefficient d'absorption du milieu varie peu par rapport à sa valeur non saturée. Par contre si $I \gg I_{\mathrm{A}}$, l'onde lumineuse a pratiquement réalisé 
l'égalité des populations entre niveaux supérieur et inférieur pour reprendre l'image simple à deux niveaux et le milieu se comporte comme un milieu transparent.

Pour analyser comment l'énergie peut se concentrer sous la forme d'une impulsion très courte dans la cavité d'un laser contenant un absorbant saturable, on étudie tout d'abord l'évolution dans la cavité d'une impulsion très énergétique initialement longue qui effectue des aller-retour dans la cavité, traversant de nombreuses fois le milieu amplificateur et l'absorbant saturable. On cherchera ensuite comment une telle impulsion peut être initiée.

On suppose que l'impulsion longue, symétrique, pénètre, en premier, dans l'absorbant saturable (Fig. 9) ; durant le temps de montée de l'impulsion, l'absorbant, initialement non saturé, absorbe une grande partie de l'intensité incidente. Mais, ce faisant,

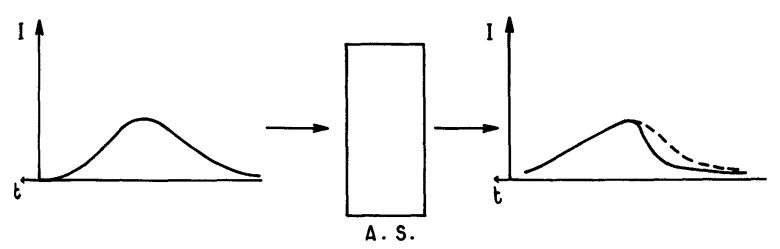

Fig. 9. - Diminution du temps de montée de l'impulsion par traversée de l'absorbant saturable.

il se sature complètement si l'intensité de l'impulsion est suffisante : son coefficient d'absorption devient nul. Comme il met ensuite un certain temps $T_{\mathrm{A}}$ pour retrouver l'équilibre des populations, si $T_{\mathrm{A}}$ est supérieur à la durée de l'impulsion, toute la queue de celle-ci traverse l'absorbant sans déformation. On constate donc que la traversée de l'absorbant saturable a raccourci son temps de montée.

Au même titre que l'absorbant, le milieu amplificateur se sature (Fig. 10). En l'absence d'onde son gain a une valeur $G_{0}$ déterminée par un certain équilibre de populations; l'onde lumineuse en modifiant cet équilibre tend à diminuer ce gain. Au fur et à mesure que l'intensité $I$ de l'onde augmente, le gain passe de $G_{0}$ à pratiquement 1 pour $I \gg I_{\mathrm{G}}$; le milieu

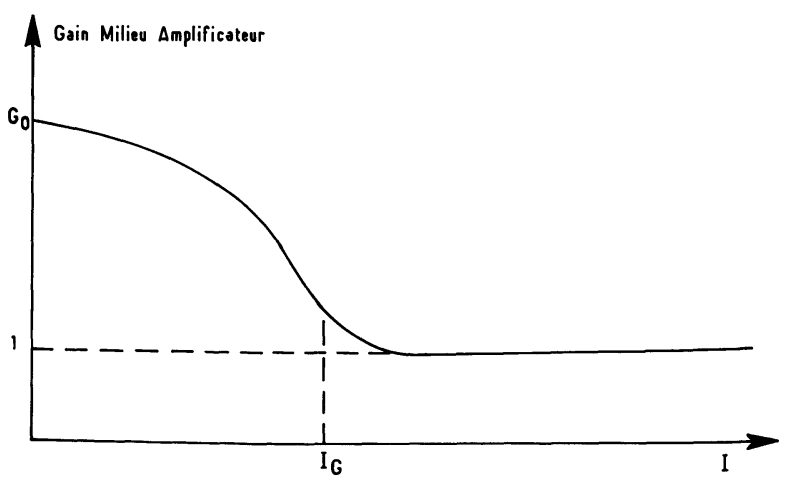

Fig. 10. - Saturation du gain du milieu amplificateur. devient transparent. $I_{G}$, qui est une caractéristique du milieu amplificateur, représente l'intensité de saturation de ce milieu qui a un comportement vis-à-vis de l'impulsion assez similaire à celui de l'absorbant saturable.

Lorsque l'impulsion dissymétrique sortant de l'absorbant pénètre dans le milieu amplificateur, celui-ci, initialement non saturé, amplifie beaucoup le front de l'impulsion. Il se sature alors progressivement et complètement si l'intensité de l'impulsion est suffisante. Comme il met ensuite un certain temps $T_{\mathrm{G}}$ à retrouver son gain $G_{0}$, la queue de l'impulsion traverse sans déformation le milieu amplificateur et va disparaître après un certain nombre de passages dans la cavité puisqu'elle subit les pertes dues au résonateur sans pouvoir bénéficier du gain du milieu amplificateur. Le temps de descente de l'impulsion est alors raccourci (Fig. 11). Le bilan global des deux traversées est une amplification de l'impulsion accompagnée d'un rétrécissement.

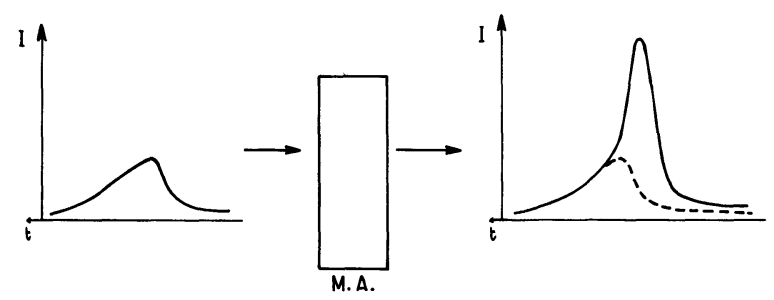

Fig. 11. - Amplification du centre de l'impulsion par le milieu amplificateur.

Le raisonnement précédent est cependant trop schématique. On a affirmé assez gratuitement que l'impulsion s'amplifiait et se rétrécissait alors que ceci dépend de la façon dont se réalisent les saturations respectives de l'absorbant et de l'amplificateur. Il faut analyser cette cinétique de manière un peu plus détaillée. Si l'on désire que le centre de l'impulsion soit amplifié, il faut qu'il traverse le milieu absorbant après que celui-ci ait été saturé, et le milieu amplificateur avant qu'il ne le soit complètement, de telle sorte que le gain global soit supérieur à l'unité. Ces conditions imposent que le milieu absorbant se sature avant le milieu amplificateur, c'est-à-dire que l'on ait la relation $I_{\mathrm{A}}<I_{\mathrm{G}}$. On désire en outre que le front de l'impulsion subisse globalement une absorption. Si l'on suppose que, lorsque l'impulsion commence à pénétrer dans les deux milieux, ceux-ci ont retrouvé leurs populations d'équilibre, perturbées lors du précédent passage, le front de l'impulsion voit un gain $G_{0}$ et une transmission $t_{0}$ non saturés. Or le produit $G_{0} t_{0}$ est obligatoirement supérieur à 1 ; en effet, s'il n'en était pas ainsi la condition d'oscillation ne pourrait être satisfaite et le laser ne démarrerait pas. Donc, si les temps $T_{\mathrm{A}}$ et $T_{\mathrm{G}}$ sont tous les deux très inférieurs à $T=\frac{2 L}{c}$, le front de l'impulsion se trouve fortement ampli- 
fié, comme le centre, et l'on n'obtient pas le rétrécissement attendu. Pour obtenir une absorption globale du front, il est nécessaire que le temps $T_{\mathbf{G}}$ soit tel que le milieu amplificateur n'ait pas le temps de revenir complètement à son état d'équilibre pendant l'aller-retour $T$ de l'impulsion, alors que l'absorbant saturable a retrouvé sa transparence minimum $t_{0}$; soit la condition $T_{\mathrm{G}} \simeq T \gg T_{\mathrm{A}}$ [11].

$\mathrm{Si}$ la dernière condition est satisfaite en même temps que la précédente : $I_{\mathrm{A}}<I_{\mathrm{G}}$, l'impulsion, à chaque aller-retour dans la cavité (Fig. 12) s'amplifie et se raccourcit. C'est la phase transitoire de sa construction pendant laquelle l'impulsion raccourcit.

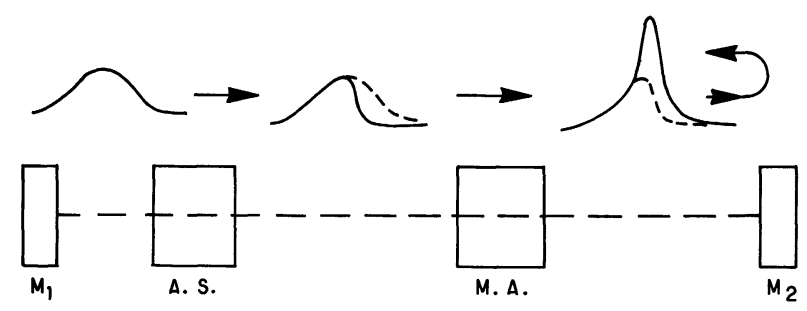

Fig. 12. - Evolution de l'impulsion à chaque aller-retour dans la cavité laser.

Il faut maintenant rechercher si l'on peut arriver à un régime stationnaire. Pour cela deux conditions : l'énergie contenue dans l'impulsion et sa durée doivent se retrouver égales à elles-mêmes, à l'état stationnaire, après un aller-retour dans la cavité. La première de ces deux conditions peut être trouvée si l'on extrapole l'évolution temporelle de l'impulsion précédemment amorcée : au fur et à mesure que l'énergie dans l'impulsion croît, les milieux amplificateur et absorbant atteignent leur saturation de plus en plus vite lors de son passage ; l'énergie apportée à l'impulsion par le milieu amplificateur, initialement très supérieure à celle dissipée dans l'absorbant, tend alors vers la somme de celle-ci et de celle perdue lors d'un aller et retour dans le résonateur; l'énergie contenue dans l'impulsion tend vers un régime stationnaire [12].

Cependant, dans le modèle précédent, l'impulsion devrait continuer à se raccourcir en raison de la saturation plus rapide de l'absorbant saturable; on ne tendrait pas vers le régime stationnaire de la durée de l'impulsion qui a été expérimentalement observé. Nous allons montrer que la durée de l'impulsion est alors limitée par les phénomènes de dispersion présents à l'intérieur de la cavité [13]. Cette dispersion n'est pas uniquement due aux éléments tels que prisme ou autres éléments disperseurs, mais aussi, bien entendu, aux milieux amplificateurs et absorbants ; elle conduit à attribuer aux différentes composantes spectrales de l'impulsion des temps de transit $T$ différents à travers chacun des éléments constituant l'oscillateur. De ce fait, au cours d'un aller et retour dans la cavité l'impulsion a tendance à s'étaler, les plus basses fréquences se retrouvant en tête et les plus élevées en fin de l'impulsion généralement. Le régime stationnaire sera alors atteint lorsque l'élargissement causé par la dispersion sera juste compensé par le rétrécissement apporté par l'ensemble milieu amplificateur absorbant saturable.

Si l'on se reporte dans l'espace des fréquences on doit remarquer qu'il n'y a pas a priori de raisons pour que la largeur minimale de l'impulsion corresponde à un blocage en phase de la totalité des modes présents dans la bande de gain de l'oscillateur. On peut très bien rencontrer la situation suivante : la bande de gain est beaucoup plus large que la largeur spectrale associée à l'impulsion la plus courte pouvant apparaître dans la cavité. Cette situation est très courante dans les lasers à colorants, pour lesquels la largeur spectrale importante du gain laisse espérer des impulsions très courtes qu'il n'est pas possible d'obtenir à cause des dispersions trop importantes inhérentes au système. On peut par exemple obtenir une bande de gain de l'ordre de $30 \AA$, sans pouvoir obtenir d'impulsions inférieures à 1 ps. On a pu montrer (dans le cas de cavités de type Bell Labs) que, dans ces conditions, seule une bande de fréquences correspondant à la production d'impulsions de 1 ps est à modes bloqués $(\simeq 5 \AA)$ et que cette bande de fréquences se déplace dans le temps sur les $30 \AA$ de la bande de gain. L'oscillateur fournit alors des impulsions de 1 ps dont la fréquence centrale n'est pas fixe mais varie sur la largeur de la bande de gain.

Les impulsions les plus courtes que l'on puisse obtenir actuellement étant de l'ordre de $0,3 \mathrm{ps}$, on est donc amené à la situation paradoxale suivante dans le cas des lasers à colorant continus : on réduit la bande de gain de ces lasers à $10 \AA ̊$ de telle sorte que tous les modes longitudinaux participent à la construction de l'impulsion, et que cette dernière présente alors une fréquence centrale bien définie.

Il reste à établir comment peut être initiée l'impulsion. En raison des couplages existant obligatoirement dans une cavité laser entre modes longitudinaux, il existe toujours des fluctuations d'intensité. Ces fluctuations sont amplifiées par l'ensemble milieu amplificateur-absorbant saturable, comme le suggèrent leurs propriétés précédemment développées, pour donner naissance à un ensemble d'impulsions plus ou moins intenses et longues. La cinétique que nous avons décrite conduit alors à la sélection et à la compression d'une seule de ces impulsions [14].

Remarque. - Le processus précédent de formation d'une impulsion à partir d'un milieu amplificateur lent $\left(T_{\mathrm{G}} \ll \tau\right)$ et d'un absorbant saturable lent $\left(T_{\mathrm{A}} \ll \tau\right)$ s'applique aux lasers à colorant mais ne s'applique pas aux lasers à solide. Dans ce dernier cas, le temps de retour à l'équilibre du milieu amplificateur est si grand $\left(T_{\mathrm{G}} \ll T\right)$, que seul l'absorbant saturable participe à la formation de l'impulsion : c'est lui qui doit atténuer à la fois le front et la queue de l'impulsion. On doit alors choisir un absorbant 
saturable rapide, puisque la durée de l'impulsion sera du même ordre de grandeur que son temps de retour à l'équilibre $\left(T_{\mathrm{A}} \simeq \tau\right)$. En outre le temps mis pour la compression de l'impulsion est beaucoup plus long que celui qui vient d'être décrit : le régime stationnaire s'établit beaucoup plus lentement.

3.3.2 Réalisations. - Comme illustration du blocage passif on donne ci-dessous quelques réalisations de lasers à colorant modes bloqués. La première est un laser pompé par flash (Fig. 13) mis en œuvre par Arthurs et autres [15] à Imperial College à Londres.

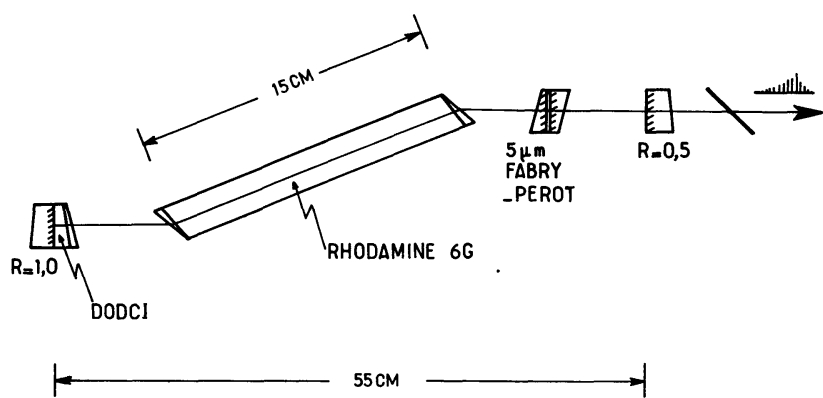

Fig. 13. - Laser à colorant modes bloqués pompé par flash (d'après Arthurs et al. [15]).

Le milieu amplificateur est constitué par le colorant le plus classique la Rhodamine 6 G (R6G), l'absorbant saturable est un autre colorant en solution qui a les caractéristiques convenables par rapport à la R6G : iodure de diéthyloxadicarbocyanine (DODCI). La largeur de bande a été volontairement limitée, pour permettre un meilleur blocage comme il a été décrit plus haut, grâce à un Fabry-Pérot. La durée des impulsions obtenues est de l'ordre de quelques ps, le temps entre impulsions de 20 ns (fréquence de répétition $50 \mathrm{MHz}$ ), la durée du train d'impulsions de l'ordre de la $\mu \mathrm{s}$. Les puissances crêtes atteignent plusieurs MW ; notons en outre qu'il est aisé d'amplifier avec de bons rendements ce train d'impulsions en utilisant des amplificateurs qui ne sont pas autre chose que des cellules de colorant (R6G) pompés en synchronisme avec le laser.

On peut alors atteindre des puissances de l'ordre du GW. La bande d'accord de tels lasers à colorant est large : en utilisant plusieurs associations colorant amplificateur-absorbant saturable [1] on peut couvrir le domaine $5800-8000 \AA$ de plus les puissances atteintes permettent d'obtenir de très bons rendements en génération de second harmonique dans des cristaux et de couvrir, avec encore beaucoup de puissance le domaine 2900-4000 Å. L'inconvénient des lasers à colorant pulsés à modes bloqués est celui de tous les lasers à colorant pulsés par flash : une assez mauvaise localisation du milieu amplificateur entraîne une reproductibilité difficile à obtenir d'un flash à l'autre.

Cet inconvénient est éliminé lorsque l'on s'intéresse aux lasers à colorant continus à modes bloqués, de conception plus récente. Deux équipes ont développé parallèlement ce type de lasers à partir de 72, l'une au laboratoire de la Bell Telephone, l'autre initialement à Belfast puis à Imperial College à Londres. Le montage d'Ippen, Shank et Dienes [16] est construit à partir de la cavité à trois miroirs à compensation d'astigmatisme qu'ils avaient proposée pour le laser à colorant continu en régime libre [17] et maintenant adoptée pour la quasi-totalité des réalisations dans ce domaine. On donne en figure 14 la version un peu sophistiquée de ce montage [18].

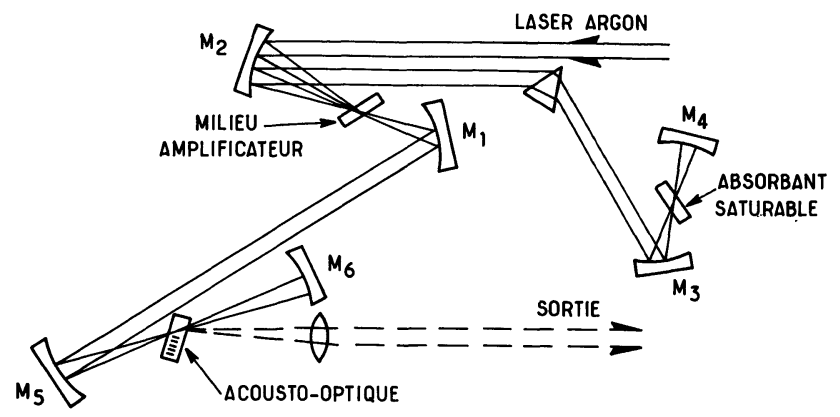

Fig. 14. - Laser à colorant continu modes bloqués par absorbant saturable (d'après E. P. Ippen et al. [18]).

Le problème posé par l'utilisation d'un absorbant saturable dans un laser continu est celui de la densité d'énergie à atteindre dans le milieu absorbant pour le saturer convenablement; en raison des faibles intensités mises en jeu en régime continu on est tenu de concentrer le faisceau à l'intérieur de l'absorbant sur des taches d'une quinzaine de $\mu \mathrm{m}$ de diamètre (même ordre de grandeur que la tache du faisceau dans le milieu amplificateur). Ceci est obtenu en remplaçant le miroir plan $\mathbf{M}_{3}$ de la cavité à trois miroirs, par un miroir $\mathbf{M}_{3}$ de faible rayon de courbure $(10 \mathrm{~cm})$, désaxé, qui concentre le faisceau sur un jet d'absorbant saturable placé à son foyer et au centre de courbure du miroir $\mathbf{M}_{4}$. L'astigmatisme apporté par le miroir $\mathbf{M}_{3}$ est compensé par celui de la lame d'absorbant placée à l'angle de Brewster. Ce dispositif a fonctionné dans sa première version sans la branche composée des miroirs $\mathbf{M}_{5}$ et $\mathbf{M}_{6}$, le miroir $\mathbf{M}_{1}$ fermant alors la cavité. Le couplage avec l'extérieur était obtenu grâce à la faible transparence de l'un des miroirs (par exemple $\mathbf{M}_{4}$ ). L'énergie obtenue alors était relativement modeste car seul un fonctionnement de ce laser près de son seuil conduisait à une stabilité convenable du blocage des modes d'une part; d'autre part les exigences d'un régime continu imposaient un couplage faible avec l'extérieur (transparence du miroir de sortie de quelques pour cent, par exemple). L'introduction d'une nouvelle branche dans la cavité, comprenant les miroirs $\mathbf{M}_{5}$ et $\mathbf{M}_{6}$ et l'extracteur de cavité (cavity dumper), permet d'améliorer considérablement ce couplage. L'extracteur de cavité est en réalité un déflecteur acousto-optique qui joue un rôle d'inter- 
rupteur commandé : à des instants contrôlés par l'onde acousto-optique que l'on envoie dans le cristal, il dévie l'impulsion qui transite dans la cavité et l'envoie à l'extérieur sur une lentille compensatrice de la divergence du faisceau. L'utilisation de cet extracteur présente un double avantage :

- L'énergie que l'on trouve dans l'impulsion ainsi extraite est approximativement celle existant dans la cavité du laser soit, par exemple 50 fois plus importante que celle que l'on peut obtenir avec un miroir de sortie de $2 \%$ de transmission.

- On peut faire varier la fréquence d'extraction des impulsions en commandant l'acousto-optique : on peut ainsi obtenir des taux de répétition allant de 0 (1 seule impulsion) à $1 \mathrm{MHz}(1$ impulsion toutes 'es $\mu \mathrm{s})$. Cependant, on ne peut atteindre, avec un tel montage, la fréquence de répétition $(\simeq 100 \mathrm{MHz})$ du montage à miroir transparent, puisqu'avant d'extraire l'impulsion de la cavité il faut laisser le régime stationnaire s'établir dans celle-ci. L'énergie dans une impulsion que l'on peut atteindre avec un tel système est de l'ordre de $5 \times 10^{-9} \mathrm{~J}$, sur une plage d'accord en longueurs d'onde qui est celle de l'association R6G-DODCI soit : 5 900-6 $200 \AA$. La puissance crête correspondante dépend de sa durée $\tau$ : on obtient couramment $\tau$ de l'ordre de 1 ps. Cependant si l'on désire obtenir des impulsions très nettement subpicosecondes on est amené à comprimer l'impulsion sortant de cette source.

Comme on l'a précédemment remarqué, en raison des éléments dispersifs contenus dans la cavité, les différentes composantes spectrales de l'impulsion ne sont pas également réparties dans son enveloppe temporelle : les plus basses fréquences sont par exemple dans le front de l'impulsion, les fréquences plus élevées en queue [18]. Pour comprimer cette impulsion il suffit de compenser le décalage temporel des différentes fréquences; l'utilisation judicieuse d'une paire de réseaux proposée par Treacy [19] conduit en particulier, à ce résultat. On obtient alors après compression de l'impulsion, que l'on fait suivre d'un filtrage spectral permettant d'éliminer les composantes qui n'ont pas été rattrapées par la compression, des durées $\tau$ aussi courtes que 0,3 ps [18]. Les puissances crêtes correspondantes sont de l'ordre de plusieurs $\mathrm{kW}$.

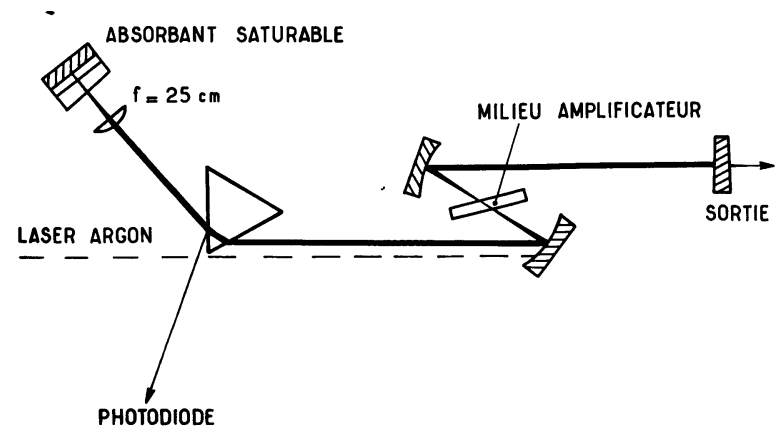

Fig. 15. - Laser à colorant continu modes bloqués par absorbant saturable (d'après I. S. Ruddock et al. [20]).
Des performances similaires ont été obtenues à Imperial College [20] en utilisant un montage quelque peu différent (Fig. 15). La concentration du faisceau dans l'absorbant saturable est réalisée au moyen d'une lentille, l'absorbant saturable étant placé directement au contact du miroir fermant la cavité. Cette position de l'absorbant saturable dans la cavité permet d'obtenir une bonne stabilité du régime permanent d'impulsions, même lorsque le laser fonctionne nettement au-dessus du seuil. On note aussi que la durée de l'impulsion est très sensible à l'épaisseur de la cellule dans laquelle circule le DODCI.

4. Détermination des durées des impulsions. - Si la mesure des énergies contenues dans les impulsions picosecondes ne pose pas de problèmes spécifiques et peut être faite avec des détecteurs classiques, thermiques par exemple, la mesure de leur durée a nécessité le développement de nouvelles techniques. En effet, actuellement les temps de montée des photodiodes les plus rapides sont encore de l'ordre de quelques dizaines de picosecondes; néanmoins on peut espérer dans un proche avenir disposer de photodétecteurs beaucoup plus rapides dont l'emploi pourra concurrencer les méthodes que l'on va décrire brièvement.

4.1 LA CAMÉRA A BALAyAge. - Elle constitue le seul dispositif permettant une mesure directe linéaire de la forme et donc de la durée d'une impulsion [21]. Le principe en est simple : les photoélectrons émis par une photocathode sous l'influence des photons de l'impulsion sont déviés par un champ électrique linéairement croissant initié à partir d'un signal donné par l'impulsion elle-même. La déviation des électrons est directement proportionnelle au temps séparant leur émission par la photocathode de l'instant de déclenchement de la rampe ; la trace qu'ils donnent sur l'écran phosphorescent est donc l'image directe de la distribution temporelle de l'intensité de l'impulsion. La réalisation est cependant délicate en raison, particulièrement, de la dispersion des temps de transit de photoélectrons émis au même instant. La technologie limite encore son utilisation aux mesures de durées $\tau>1$ ps. En outre, la caméra à balayage constitue un investissement important que peu de petites équipes arrivent à réaliser.

4.2 GÉNÉRATION DU SECOND HARMONIQUE. - Au contraire de la précédente, les deux techniques qui suivent, outre la possibilité qu'elles donnent de faire des mesures de durées d'impulsions subpicosecondes, peuvent être mises en œuvre sans moyens importants. La première, la plus importante concernant les mesures d'impulsions subpicosecondes, utilise la génération de second harmonique dans un cristal non linéaire (SHG). Proposée en 1966 [22], elle a été utilisée pour la première fois dans le cas des lasers à colorant continus à modes bloqués par A. Dienes, E. P. Ippen, 
C. V. Shank [23]. Le dispositif expérimental est donné en figure 16. Le principe en est le suivant : le train d'impulsions délivré par la source est partagé en deux faisceaux de même intensité par une lame séparatrice de $50 \%$ de transmission. Les deux faisceaux, après avoir suivi des chemins optiques différents, sont recombinés et focalisés dans un cristal non linéaire. Le réflecteur, dans l'un des bras de l'interféromètre, peut être déplacé parallèlement à lui-même, changeant ainsi le chemin optique de l'un des faisceaux par rapport à l'autre. Lorsque celui-ci est tel que les impulsions des deux faisceaux se recouvrent exactement au niveau du cristal, la génération de second harmonique mesurée par le détecteur est maximale.

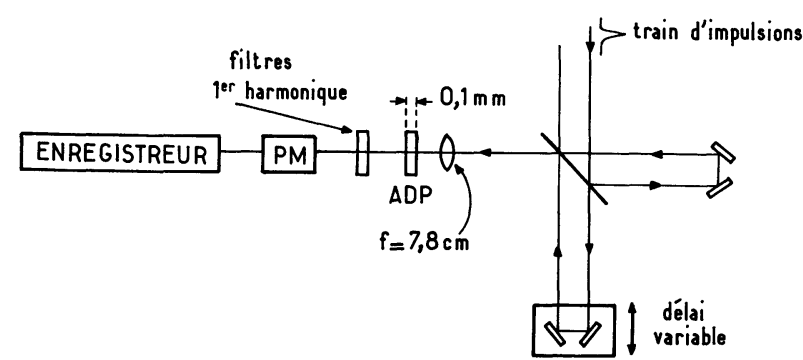

Fig. 16. - Dispositif expérimental pour une mesure de génération de second harmonique (d'après A. Dienes et al. [23]).

En déplaçant le miroir de l'interféromètre par rapport à cette position, on décale les impulsions l'une par rapport à l'autre, le signal de SHG diminue jusqu'à tendre vers une constante pour les deux impulsions ne se recouvrant plus du tout. Le déplacement linéaire dans le temps du miroir permet alors d'obtenir sur un enregistreur à défilement du papier également linéaire, recevant la sortie du photomultiplicateur, un signal fonction à chaque instant du recouvrement des deux impulsions ; la largeur du signal utile correspond donc à une mesure indirecte de la longueur $c \tau$ de l'impulsion dans l'espace. On a remplacé la mesure d'un temps $\tau$, par la mesure d'un déplacement en mettant à profit la connaissance de la vitesse $c$ de propagation de l'impulsion. En fait, le lien entre signal obtenu et forme de l'impulsion est assez complexe : le signal de second harmonique $E_{2 \omega}$ qui prend naissance dans le cristal est proportionnel au carré du champ $E_{\omega}$ qu'il reçoit, $E_{\omega}$ étant lui-même égal à la somme des champs dans chacun des faisceaux

$E_{\omega}=E_{1}(t)+E_{2}(t-\theta)$

où $\theta$ désigne le retard de l'une des impulsions par rapport à l'autre. Le détecteur est lui sensible à la moyenne dans le temps du carré du champ à la fréquence $2 \omega, E_{2 \omega}$. On montre que le signal $s(\theta)$ enregistré, s'exprime à partir de la fonction d'autocorrélation du second ordre $G^{2}(\theta)$ de l'intensité $I(t)$ de l'impulsion [4] :

$s=1+2 G^{2}(\theta)$ où $\quad G^{2}(\theta)=\frac{\langle I(t) I(t-\theta)\rangle}{\left\langle I^{2}(t)\right\rangle}$.
L'allure du signal $s(\theta)$ donne de précieuses indications sur la qualité du blocage des modes obtenu. En effet, dans le cas d'un blocage parfait, l'expression précédente indique un rapport 3 entre le fond continu et le maximum du signal. Si le blocage est incomplet, ce qui correspond à une impulsion présentant des fluctuations aléatoires rapides de son amplitude dans le temps, le signal ne présente plus qu'un contraste de 2 à 1 seulement, avec, en plus, un pic d'amplitude 3 caractéristique de la corrélation du bruit d'amplitude (Fig. 17).

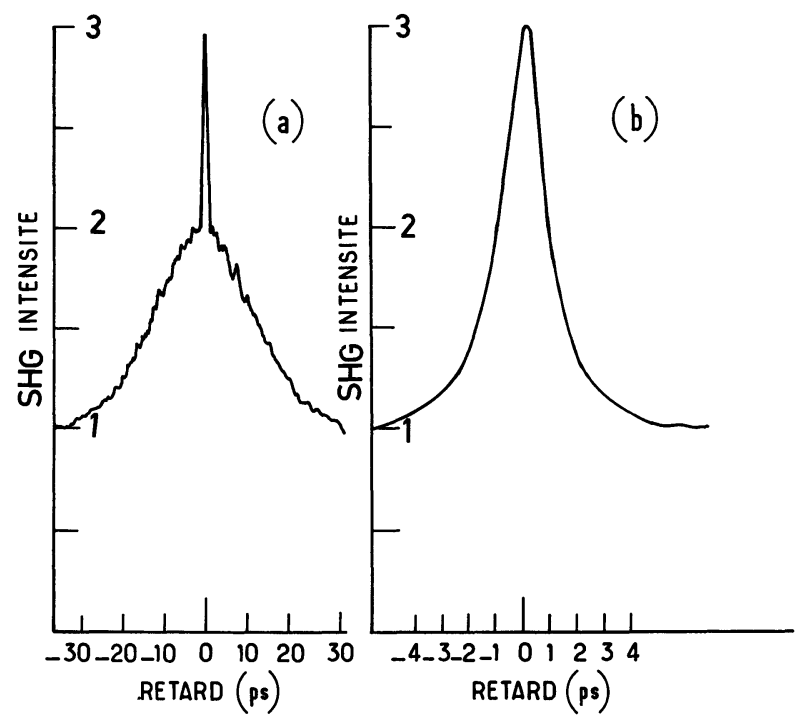

Fig. 17. - Signaux obtenus en génération de second harmonique : l) pour un blocage de modes imparfait (rapport $2 / 1+$ pic de corrélation du bruit); $b$ ) pour un bon blocage de modes (rapport 3/1).

Mais la connaissance de $s(\theta)$, même dans le cas d'un blocage parfait des modes, est insuffisante pour déterminer la forme et la durée exacte de l'impulsion. Si l'on fait l'hypothèse d'une forme analytique pour l'impulsion, on peut calculer le lien existant entre la largeur de la courbe $\Delta \theta$ de $s(\theta)$ (largeur totale à mi-hauteur) et la durée $\tau$ de l'impulsion (largeur totale à mi-hauteur de $I(t))$. Dans le cas d'une impulsion de forme gaussienne par exemple,

$\frac{\Delta \theta}{\tau} \simeq \sqrt{2}$

dans le cas d'une impulsion dont la forme est celle trouvée par Hauss [13] en $1 / c h^{2}$, ce rapport vaut 1,55. On remarque néanmoins que le facteur introduit pour tenir compte de la forme de l'impulsion varie assez peu autour de 1,5. $\Delta \theta$ donne donc une bonne idée de la valeur de $\tau$. Seule la détermination supplémentaire de fonctions d'autocorrélations $G^{n}(\theta)$ d'ordres plus élevés permettrait de remonter à la forme et à la durée exacte de l'impulsion [1].

Le relevé du spectre de l'impulsion peut également donner d'intéressantes indications; cependant, outre le fait que la bande spectrale $\Delta v$ occupée par l'im- 
pulsion n'est simplement reliée à $\tau$ que dans le cas où le blocage des modes est parfait (on dit que l'impulsion satisfait alors la transformée de Fourier) le lien entre $\Delta v$ et $\tau$ dépend lui aussi et beaucoup plus fortement de la forme de l'impulsion : $\Delta v \cdot \tau=0,441$ pour une impulsion gaussienne, 0,315 pour une impulsion en $1 / c h^{2}$. Cette mesure du spectre ne peut donc venir qu'en appui de la mesure de SHG pour confirmer le bon blocage des modes. Un inconvénient important de la méthode de SHG, est qu'elle nécessite un nombre important d'impulsions pour pouvoir obtenir la totalité de la courbe $s(\theta)$, puisque chaque point de cette courbe correspond à un retard de l'une des impulsions par rapport à l'autre et que l'on fait varier lentement ce retard. On voit par exemple que pour un temps typique d'enregistrement de $10 \mathrm{~s}$ et un écart entre impulsions de $10 \mathrm{~ns}$, le tracé de $s(\theta)$ correspond à $10^{9}$ impulsions. Si l'on peut facilement obtenir ce nombre d'événements à partir du train continu d'impulsions délivré par un laser à colorant continu modes bloqués, il n'en est pas de même lorsque l'on s'intéresse à la sortie pulsée d'un laser à solide. On préfère le plus souvent dans ce cas utiliser une seconde méthode non linéaire : la fluorescence à deux photons.

4.3 Fluorescence A deux photons. - Proposée par Giordmaine [24], cette technique consiste (Fig. 18) à envoyer dans des directions opposées à l'intérieur d'un colorant fluorescent en solution, les deux faisceaux collinéaires obtenus grâce à une lame séparatrice $50 \%$ à partir de la sortie du laser. Le colorant est choisi de manière à n'absorber qu'au double de la fréquence de la source. Le rendement de cette fluorescence à deux photons est maximal au point de la cuve où il y a recouvrement des deux impulsions. La photographie de la fluorescence donne un signal dont chaque point correspond à un point de la courbe $s(\theta)$ précédente. L'avantage réside en ce qu'une seule impulsion peut donner l'ensemble du signal puisque la plaque photographique donne une image directe du recouvrement des deux impulsions. L'analyse du signal est similaire à celle de SHG, l'intensité reçue par la plaque en un point

$\begin{array}{ccc}\text { Colorant continu } & \begin{array}{c}\text { Colorant continu } \\ \text { absorbant saturable } \\ \text { pompés en synchro }\end{array} \\ \begin{array}{c}\text { Durée } \\ \text { d'impulsion }\end{array} & - & - \\ \begin{array}{c}\text { Puissance } \\ \text { crête }\end{array} & 0,3 \mathrm{ps} & \text { qqes ps } \\ \text { Durée } & \text { qqes kW } & \text { qqes } 100 \mathrm{~W} \\ \text { du train } & \text { continue } & \text { continue } \\ \text { Fréquence } & \text { 100 MHz ou } & 100 \mathrm{MHz} \text { ou } \\ \text { des impulsions } & \text { variable } 0-1 \mathrm{MHz} & \text { variable } 0-1 \mathrm{MHz} \\ & & \\ \text { Plage accord } & 5900-6200 & 5650-6250 \\ \lambda(\AA) & 2950-3100 & 2825-3125 \\ & \text { Génération d'Harmoniques } & (\mathrm{G} . \mathrm{H} .) \\ & (\mathrm{G} . \mathrm{H} .) & \end{array}$

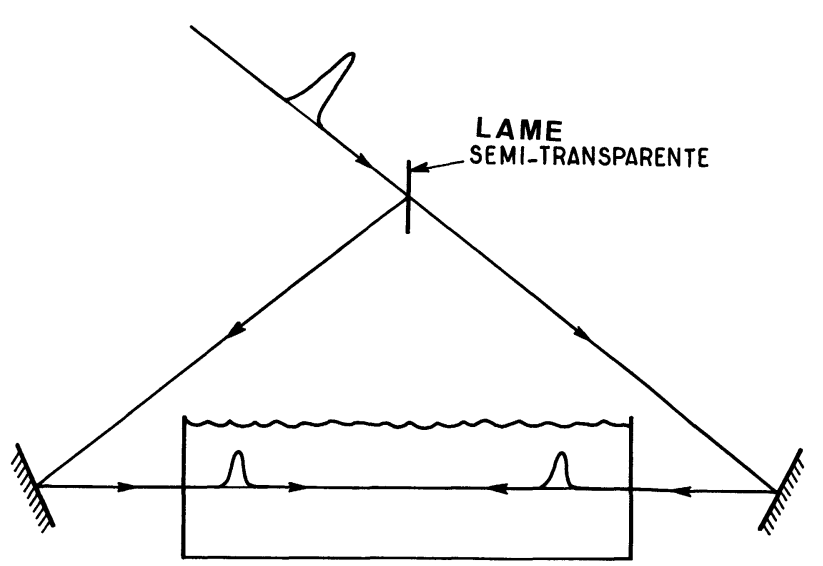

Fig. 18. - Dispositif expérimental pour une mesure de fluorescence à deux photons (d'après [1]).

donné étant proportionnelle à $1+2 G^{2}(\theta)$ comme précédemment. Cependant, des difficultés technologiques liées par exemple à un bon étalonnage du noircissement de la plaque, rendent délicate l'exploitation quantitative des résultats. On préfère généralement utiliser lorsque cela est possible la méthode de SHG.

5. Tableau comparatif des performances obtenues avec les lasers picosecondes les plus courants. - On va maintenant comparer les performances obtenues à partir des systèmes que nous venons de décrire, à celles fournies par les autres lasers picosecondes classiques. Il faut noter que les performances annoncées sont celles que l'on admet couramment comme les meilleures en début de l'année 1978. L'évolution est telle, dans ce domaine, qu'une mise à jour devrait être effectuée au bout de périodes n'excédant pas quelques mois.

On remarque tout de suite que si les lasers solides à impulsions géantes n'ont pas encore de concurrent dans le domaine des très fortes énergies contenues dans les impulsions, les lasers à colorant continus à modes bloqués par absorbant saturable n'ont pas de concurrent dans le domaine subpicoseconde. Cependant, par rapport aux autres lasers à colorant

\begin{tabular}{|c|c|c|}
\hline $\begin{array}{c}\text { Colorant pulsé } \\
\text { absorbant saturable } \\
+\end{array}$ & $\begin{array}{c}\text { Colorant pulsé } \\
\text { pompé en synchro } \\
-\end{array}$ & $\begin{array}{l}\text { Verres dopés ou rubi } \\
\text { absorbant saturable } \\
\end{array}$ \\
\hline 5 ps & $10 \mathrm{ps}$ & 5 ps \\
\hline $\begin{aligned} & \text { qqes } 10 \mathrm{MW} \\
+ & \text { Ampli } \rightarrow 1 \mathrm{GW}\end{aligned}$ & $1 \mathrm{MW}$ & $\begin{array}{c}\text { qqes } 100 \mathrm{MW} \\
+ \text { Ampli } \rightarrow 1 \mathrm{TW}\end{array}$ \\
\hline$\simeq 1 \mu \mathrm{s}$ & $\simeq 0,1 \mu \mathrm{s}$ & $\simeq 0,1 \mu \mathrm{s}$ \\
\hline qqes $100 \mathrm{MHz}$ & qqes $100 \mathrm{MHz}$ & qqes $100 \mathrm{MHz}$ \\
\hline & & 10600 \\
\hline $5800-8000$ & Visible & 5300 \\
\hline $\begin{array}{c}2900-4000 \\
\text { (G.H.) }\end{array}$ & proche UV & 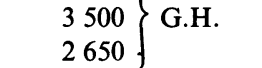 \\
\hline
\end{tabular}


la plage d'accord de ces derniers est relativement restreinte. De nombreuses équipes se sont récemment intéressées au problème de l'élargissement de cette plage.

6. Récents développements concernant l'élargissement de la plage d'accord des lasers à colorant continus à modes bloqués par absorbant saturable. - Le problème de limitation de la plage provient de ce que peu de couples milieu amplificateur-absorbant saturable conduisent à un fonctionnement modes bloqués convenable en régime continu. Seule pour l'instant la R6G associée à quelques absorbants saturables (DODCI, fluoresceine ou crésyl-violet) a été utilisée pour le milieu amplificateur.

En attendant que d'autres associations soient proposées, nous avons essayé de mieux utiliser l'association la plus classique R6G-DODCI. Nous avons développé une source fournissant deux trains d'impulsions picosecondes synchrones, à deux longueurs d'onde séparément accordables [25]. Ce laser, dont le montage est représenté en figure 19, est composé de deux résonateurs construits à partir du même milieu amplificateur et du même prisme sélecteur. On reconnaît dans le résonateur I les éléments caractérisant un laser à colorant à modes bloqués par absorbant saturable; le résonateur II correspond à un laser à colorant continu classique.

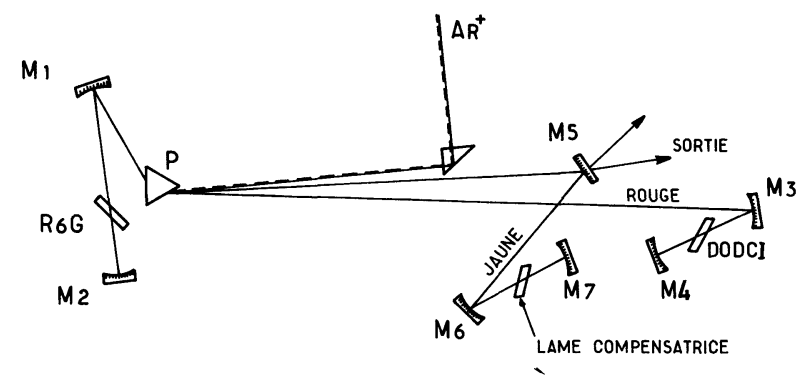

Fig. 19. - Source laser fournissant deux trains d'impulsions picosecondes synchrones à deux longueurs d'ondes séparément accordables (d'après B. Couillaud et A. Ducasse [25]).

Le résonateur I est accordé sur une longueur d'onde visible dans le domaine rouge du spectre, le résonateur II dans le jaune. Les deux oscillateurs sont fortement couplés par l'intermédiaire du milieu amplificateur puisque ce sont les mêmes molécules qui sont susceptibles d'amplifier l'un ou l'autre des faisceaux. Ainsi, en l'absence d'absorbant saturable dans le résonateur $I$, le régime libre d'oscillations ne peut s'établir dans les deux cavités à la fois : selon leurs pertes relatives, soit l'un, soit l'autre des oscillateurs fonctionne; si l'on coupe l'oscillation de plus grand gain, la seconde oscillation qui n'est plus étouffée par l'autre peut alors apparaître.

Lorsque maintenant on fait circuler dans le résonateur I l'absorbant saturable, le laser I de longueur $L_{1}$
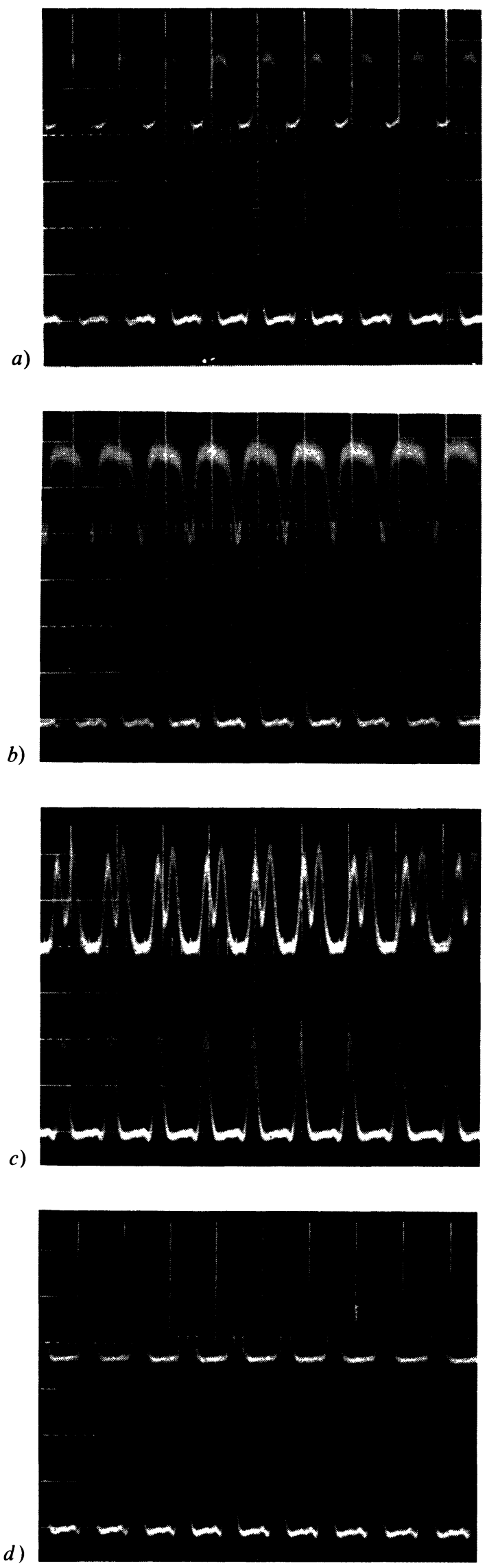

Fig. 20. - Oscillogrammes reproduisant l'évolution des impulsions lorsque $L_{\mathrm{II}}$ varie au voisinage de la valeur $L_{1}$ (d'après B. Couillaud et A. Ducasse [25]). 
fonctionne en régime à modes bloqués. Le passage de l'impulsion correspondante dans le milieu amplificateur module son gain à la fréquence $c / 2 L_{\mathrm{I}}$. Cette modulation du gain joue alors un rôle de blocage actif pour les modes du résonateur II si l'on ajuste la longueur $L_{\mathrm{II}}$ à une valeur voisine de $L_{\mathrm{F}}$.

Cet effet a bien été observé (Fig. 20). L'oscillateur I rouge fournit le train d'impulsions picosecondes représenté par la trace du bas de tous les oscillogrammes. On fait varier $L_{\mathrm{II}}$ au voisinage de la valeur $L_{\mathrm{I}}$ et l'on observe la sortie de l'oscillateur jaune; on peut alors relever la cinétique de formation de l'impulsion jaune : la sortie de I est d'abord sinusoïdale ; puis on observe des absences d'impulsions ou impulsions noires qui correspondent à une émission continue interrompue périodiquement (période $C / 2 L$ ), la durée de l'interruption étant inférieure à 1 ns. Une génération de signaux de cette forme n'avait jamais encore été rapportée et pourrait avoir d'intéressantes applications. Lorsque l'on continue à rapprocher la longueur $L_{\mathrm{II}}$ de $L_{\mathrm{l}}$, on observe ensuite sur la sortie jaune des doubles impulsions, puis pour $L_{\mathrm{II}} \simeq L_{\mathrm{I}}$ [26], un train d'impulsions pour chaque sortie. Les largeurs d'impulsions présentées sur les oscillogrammes sont bien entendu celles du système de détection. L'étude de leurs caractéristiques est actuellement en cours. On a ainsi pu obtenir un train d'impulsions accordable de 6100 à $6200 \AA$, le deuxième train synchrone étant séparément accordable de 5650 à $5900 \AA$; la plage d'accord est ainsi étendue vers le jaune.

Presque simultanément à l'Université de Berkeley, Yasa, Dienes, Whinnery [27] ont obtenu deux trains d'impulsions synchrones à deux longueurs d'onde différentes en utilisant une méthode tout à fait différente. Le montage représenté en figure 21 est celui du laser classique avec la R6G comme milieu amplificateur et le crésyl-violet comme absorbant saturable. Cependant, les auteurs ont pu montrer que le crésyl-violet, qui joue un rôle d'absọrbant saturable pour l'impulsion qui prend naissance dans la R6G, devient amplificateur pour des longueurs d'ondes plus longues après le passage de cette impulsion.

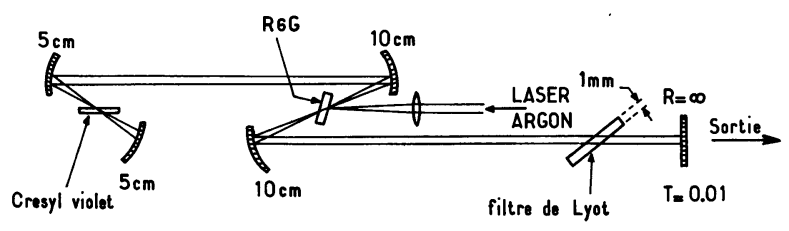

Fig. 21. - Laser à colorant continu modes bloqués fournissant deux trains d'impulsions synchrones (d'après Yasa et al. [27]).

Il joue alors à son tour le rôle d'un milieu amplificateur dont le gain est modulé à la fréquence $c / 2 L$; cette modulation active conduit donc à la génération d'une nouvelle impulsion de plus grande longueur d'onde se déplaçant à la suite de la première dans la même cavité. La première impulsion raccourcie par la présence de la seconde est subpicoseconde $(0,5 \mathrm{ps})$; la durée de l'impulsion qui prend naissance dans le crésyl-violet est de l'ordre de 1 ps. Les pics de puissance des impulsions des trains sortant de la cavité grâce à la transparence d'un des miroirs sont respectivement de l'ordre de $100 \mathrm{~W}$ pour le premier train, $10 \mathrm{~W}$ pour le second. Le domaine d'accord du premier train est autour de $6000 \AA$, cependant que le second est accordable autour de $6400 \AA$. La plage a été étendue vers les grandes longueurs d'onde.

Tout récemment Jain, Heritage [28] ont repris les idées des deux précédents montages pour parvenir à une nouvelle méthode originale de génération d'impulsions synchrones. Ils ont particulièrement montré l'intérêt du pompage synchrone par rapport à la méthode passive de blocage des modes ; ce type de montage pourrait avoir un développement important dans le proche avenir.

Il faut enfin noter les performances extraordinaires obtenues aux Laboratoires de la Bell Telephone : une impulsion picoseconde extraite d'un laser à colorant continu modes bloqués par absorbant saturable de durée $0,5 \mathrm{ps}$, puissance crête quelques $\mathrm{kW}$ passe à travers trois amplificateurs et donne une impulsion, toujours de 0,5 ps et de $400 \mathrm{GW}$ de puissance crête (énergie contenue dans l'impulsion $0,2 \mathrm{~J}$ ). Cette impulsion est envoyée dans une cellule d'eau où par effet Raman, elle éclate en fréquences sur tout un spectre s'étendant de $2200 \AA$ à $2 \mu \mathrm{m}$ conservant sa durée de 0,5 ps. Des filtres appropriés permettent alors d'isoler des impulsions de 0,5 ps n'importe où dans cette bande spectrale. Les puissances crêtes obtenues sont de l'ordre de quelques $\mathrm{kW}$. Un tel montage, n'est cependant pas à la portée de n'importe quelle équipe tant par l'investissement qu'il suppose que par la complexité de sa mise en œuvre.

On peut se rendre compte, à partir des travaux rapportés dans la dernière partie de cet article, que la production d'impulsions picosecondes est un domaine en pleine évolution, principalement en ce qui concerne le développement des systèmes utilisant des lasers à colorant continus. On a présenté à un instant donné de l'évolution, les performances atteintes, mais aussi et surtout on a essayé de donner d'une manière aussi imagée que possible les concepts de base permettant de comprendre les processus conduisant à une concentration de l'énergie dans le temps. Il est certain que, dans ce domaine aussi, des progrès restent à faire, un certain nombre de points restant obscurs. Cependant, aussi bien dans la compréhension de ces processus que dans l'évolution des techniques on a actuellement atteint un stade suffisamment avancé pour que la spectroscopie picoseconde puisse maintenant se développer. On peut prévoir que des domaines tels que la photochimie ou la biochimie où les processus de relaxation rapides sont très importants vont, dans les prochaines années, bénéficier des résultats obtenus grâce à ces nouvelles techniques. 


\section{Bibliographie}

[1] Topics in Applied Physics, Ultrashort light pulses - Picosecond techniques and Applications, Ed. S. L. Shapiro (Springer-Verlag) 1977.

[2] Topics in Applied Physics, Dye Lasers, Ed. F. P. Schäfer (Springer-Verlag) 1973.

[3] Sмith, P. W., Proc. IEEE 58 (1970) 1342.

[4] De Maria, A. J. et al., Proc. IEEE 57 (1969) 2.

[5] LeTHOKOv, V. S., J.E.T.P. 28 (1969) 562.

[6] Kogelnik, H., Li, T., Proc. IEEE 54 (1966) 1312.

[7] Sмith, P. W., Appl. Phys. Lett. V 13 (1968) 235.

[8] Auston, D. H., IEEE J. Quant. Electr. QE 4 (1968) 420.

[9] Electronique Quantique en vue des Applications, R. H. Pantell, H. E. Puthoff (Dunod) 1973.

[10a] Crowell, M. H., IEEE J. Quant. Electr. QE 1 (1965) 12.

[10b] Kuizenga, D. J., Siegman, A. E., IEEE J. Quant. Electr. QE 6 (1970) 709.

[11] New, G. H. C., Opt. Commun. V 6 (1972) 188.

[12] New, G. H. C., IEEE J. Quant. Electr. QE 10 (1974) 115.

[13] Hauss, H. A., IEEE J. Quant. Electr. QE 11 (1975) 736.

[14] Yasa, Z. A., Teschke, O., Braverman, L. W., Dienes, A Opt. Commun. 15 (1975) 354.

[15] Arthurs, E. G., Bradley, D. J., Roddie, A. G., Appl. Phys. Lett. 19 (1971) 480.
[16] Ippen, E. P., Shank, C. V., Dienes, A., Appl. Phys. Lett. V 21 (1972) 348.

[17] Kogelnik, H., Ippen, E. P., Dienes, A., Shank, C. V., IEEE J. Quant. Electr. QE 8 (1972) 373.

[18] Ippen, E. P., Shank, C. V., Appl. Phys. Lett. V 27 (1975) 488.

[19] Treacy, E. B., Phys. Lett. V 28A (1968) 34 ; Appl. Phys. Lett. V 14 (1969) 112 ; IEEE J. Quant. Electr. QE 5 (1969) 454.

[20] Ruddock, I. S., Bradley, D. J., Appl. Phys. Lett. V 29 (1976) 296.

[21] Proc. 10th Congr. High Speed Photography (A.N.R.T., Paris 1972), ARthurs, E. G., Bradley, D. J., Liddy, B., O'Neill, F., Roddie, A. G., Sibbett, W., Sleat, W. E.

[22] Maier, M., Kaiser, W., Giordmaine, J. A., Phys. Rev. Lett. V 17 (1966) 1275.

[23] Dienes, A., Ippen, E. P., Shank, C. V., Appl. Phys. Lett. V 19 (1971) 258

[24] Giordmaine, J. A., Rentzepis, P. M., Shapiro, S. L., Wecht, K. W., Appl. Phys. Lett. 11 (1967) 216.

[25] Couillaud, B., Ducasse, A., Appl. Phys. Lett. 29 (1976) 665.

[26] Scavennec, A., Opt. Commun. 17 (1976) 14.

[27] Yasa, Z. A., Dienes, A., Whinnery, J. B., Appl. Phys. Lett. 30 (1977) 24.

Yasa, Z. A., Teschke, O., Appl. Phys. Lett. 27 (1975) 446.

[28] Jain, R. K., Heritage, J. P., Appl. Phys. Lett. 32 (1978) 41. 